\title{
Staphylococcus aureus
}

\begin{tabular}{|c|c|c|c|c|c|c|c|c|c|}
\hline Duts $^{b}$ & $80 \alpha$ & $\phi$ ROSA & $\phi 71$ & $\phi$ ETA2 & $\phi P V L 108$ & $\phi$ NM3 & $\phi 85$ & $\phi 3 \mathrm{~A}$ & $\phi$ PVL-CN125 \\
\hline $80 \alpha$ & 0 & & & & & & & & \\
\hline$\phi R O S A$ & $\begin{array}{c}-0,232 \\
(0,076)\end{array}$ & 0 & & & & & & & \\
\hline$\phi 71$ & $\begin{array}{c}-0,161 \\
(0,064) \\
\end{array}$ & $\begin{array}{c}-0,210 \\
(0,062)\end{array}$ & 0 & & & & & & \\
\hline$\phi$ ETA2 & $\begin{array}{l}-0,136 \\
(0,058)\end{array}$ & $\begin{array}{c}-0,156 \\
(0,059)\end{array}$ & $\begin{array}{c}-0,113 \\
(0,053)\end{array}$ & 0 & & & & & \\
\hline$\phi P V L 108$ & $\begin{array}{l}-0,151 \\
(0,063) \\
\end{array}$ & $\begin{array}{l}-0,178 \\
(0,057)\end{array}$ & $\begin{array}{l}-0,097 \\
(0,044)\end{array}$ & $\begin{array}{l}-0,040 \\
(0,044)\end{array}$ & 0 & & & & \\
\hline$\phi N M 3$ & $\begin{array}{c}-0,179 \\
(0,073)\end{array}$ & $\begin{array}{c}-0,218 \\
(0,058)\end{array}$ & $\begin{array}{c}-0,144 \\
(0,053)\end{array}$ & $\begin{array}{c}-0,150 \\
(0,054)\end{array}$ & $\begin{array}{c}-0,113 \\
(0,045)\end{array}$ & 0 & & & \\
\hline$\phi 85$ & $\begin{array}{l}-0,043 \\
(0,045)\end{array}$ & $\begin{array}{c}-0,253 \\
(0,065)\end{array}$ & $\begin{array}{c}-0,141 \\
(0,057)\end{array}$ & $\begin{array}{l}-0,047 \\
(0,038)\end{array}$ & $\begin{array}{l}-0,111 \\
(0,049)\end{array}$ & $\begin{array}{l}-0,153 \\
(0,040)\end{array}$ & 0 & & \\
\hline$\phi 3 A$ & $\begin{array}{l}-0,269 \\
(0,078)\end{array}$ & $\begin{array}{c}-0,190 \\
(0,070)\end{array}$ & $\begin{array}{c}-0,120 \\
(0,055)\end{array}$ & $\begin{array}{c}-0,130 \\
(0,049)\end{array}$ & $\begin{array}{l}-0,006 \\
(0,037)\end{array}$ & $\begin{array}{l}-0,176 \\
(0,063)\end{array}$ & $\begin{array}{l}-0,192 \\
(0,068)\end{array}$ & 0 & \\
\hline $\begin{array}{c}\phi P V L- \\
\text { CN125 }\end{array}$ & $\begin{array}{l}-0,157 \\
(0,064)\end{array}$ & $\begin{array}{l}-0,206 \\
(0,060)\end{array}$ & $\begin{array}{l}-0,065 \\
(0,028)\end{array}$ & $\begin{array}{l}-0,094 \\
(0,051)\end{array}$ & $\begin{array}{l}-0,061 \\
(0,040)\end{array}$ & $\begin{array}{l}-0,082 \\
(0,045)\end{array}$ & $\begin{array}{c}-0,10 \\
(0,051)\end{array}$ & $\begin{array}{l}-0,116 \\
(0,056)\end{array}$ & 0 \\
\hline
\end{tabular}

\begin{tabular}{|c|c|c|c|c|c|}
\hline SaPIbov2 $^{c}$ & $\phi$ ROSA & $\phi 85$ & $80 \alpha$ & DAR3907 & PLAC6004 \\
\hline$\phi$ ROSA & 0 & & & & \\
\hline$\phi 85$ & $-0,011(0,025)$ & 0 & & & \\
\hline $80 \alpha$ & $-0,590(0,300)$ & $-0,552(0,282)$ & 0 & & \\
\hline DAR3907 & $-0,631(0,300)$ & $-0,593(0,280)$ & $-0,128(0,064)$ & 0 & \\
\hline PLAC6004 & $-0,084(0,054)$ & $-0,059(0,045)$ & $-0,581(0,287)$ & $-0,621(0,282)$ & 0 \\
\hline
\end{tabular}

\begin{tabular}{|c|c|c|c|c|c|c|c|}
\hline $\mathrm{Sri}^{\mathrm{d}}$ & A6300 & Mu50 & $\phi 85$ & 21252 & M79256 & $80 \alpha$ & $\phi 55$ \\
\hline A6300 & 0 & & & & & & \\
\hline Mu50 & $-0,098(0,069)$ & 0 & & & & & \\
\hline$\phi 85$ & $-0,089(0,056)$ & $-0,125(0,087)$ & 0 & & & & \\
\hline 21252 & $-0,372(0,190)$ & $-0,363(0,199)$ & $-0,224(0,132)$ & 0 & & & \\
\hline M79256 & $-1,284(0,684)$ & $-1,017(0,627)$ & $-0,740(0,549)$ & $-0,541(0,472)$ & 0 & & \\
\hline $80 \alpha$ & $-0,186(0,094)$ & $-0,090(0,076)$ & $-0,154(0,090)$ & $-0,287(0,161)$ & $-1,148(0,666)$ & 0 & \\
\hline$\phi 55$ & $-0,223(0,111)$ & $-0,124(0,089)$ & $-0,256(0,134)$ & $-0,460(0,239)$ & $-0,800(0,587)$ & $-0,136(0,088)$ & 0 \\
\hline
\end{tabular}

\section{Enterococcus faecalis}

\begin{tabular}{|c|c|c|c|c|}
\hline E. faecalis $^{\text {e }}$ & EF0309 & Ef11 & X98 & VC1B-1 \\
\cline { 1 - 3 } EF0309 & 0 & \multicolumn{2}{|c}{} \\
Ef11 & $-0,016(0,346)$ & 0 & 0 & 0 \\
\hline X98 & $0,115(0,294)$ & $-0,028(0,029)$ & $-1,128(0,468)$ & 0 \\
\hline VC1B-1 & $-0,115(0,493)$ & $-1,085(0,444)$ &
\end{tabular}

${ }^{a}$ The difference between the nonsynonymous and synonymous distances per site from between sequences are shown. Standard error estimate(s) are shown in parenthesis. Analyses were conducted using the modified Nei-Gojobori (assumed transition/transversion bias = 2) model [1]. Evolutionary analyses were conducted in MEGA5 [2].

${ }^{b}$ Accession numbers Dut proteins: $80 \alpha$ (YP_001285346); $\phi R o s a$ (YP_240373); $\phi 71$ (YP_240446); $\phi E T A 2$ (YP_001004294); $\phi P V L 108$ (YP_918921); $\phi N M 3$ (YP_908820); $\phi 85$ (YP_239795); $\phi 3 A$ (YP_239989); $\phi P V L-C N 125$ (YP_002939700).

${ }^{c}$ Accession numbers SaPlbov2 inducers: $\phi R O S A$ (YP_240350); $\phi 85$ (YP_239776); 80 $\alpha$ (YP_001285329); Strain DAR390 (EXY00034); Strain PLAC6004 (EVF70071).

${ }^{\mathrm{d} A c c e s s i o n}$ numbers Sri proteins: Strain A6300 (EEV76885); Strain Mu50 (BAB57032); $\phi 85$ (YP_239784); Strain 21252 (EHO92899); Strain M79256 (EXP87181); $\phi 80 \alpha$ (YP_001285336); $\phi 55$ (YP_240514).

${ }^{\text {e}}$ Accession numbers EfClv583 inducer proteins: EF0309 (AAO80172); Ef11 (YP_003358829); X98 (WP_002381619); VC1B-1(EPI33180). 